\title{
Unified viscoelasticity: \\ Applying discrete element models to soft tissues with two characteristic times
}

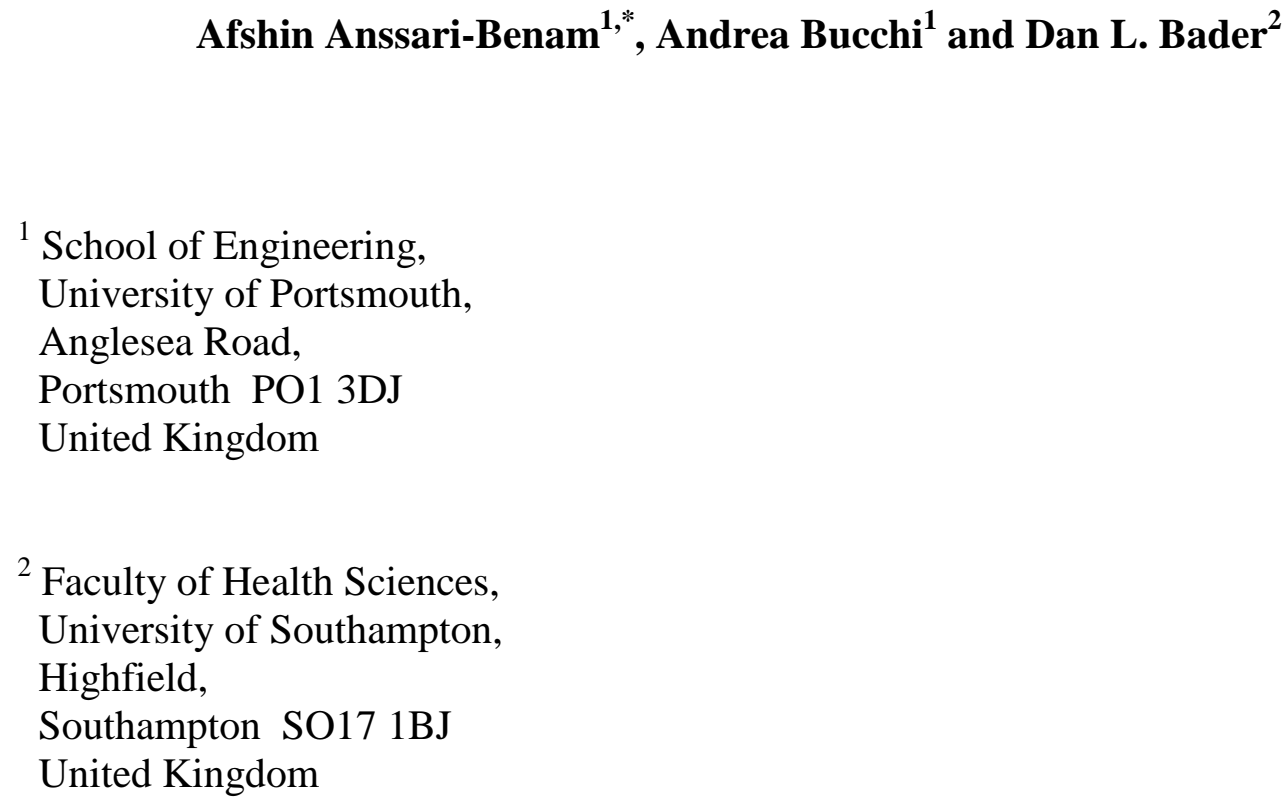

*Address for correspondence: Afshin Anssari-Benam, School of Engineering, University of Portsmouth, Anglesea Road, Portsmouth PO1 3DJ United Kingdom

Tel: $\quad$ ++44 (0)2392842187 Fax: $\quad++44$ (0)23 92842351

E-mail: afshin.anssari-benam@port.ac.uk

Word count (Introduction to conclusion): 2741 


\section{Abstract}

Discrete element models have often been the primary tool in investigating and characterising the viscoelastic behaviour of soft tissues. However, studies have employed varied configurations of these models, based on the choice of the number of elements and the utilised formation, for different subject tissues. This approach has yielded a diverse array of viscoelastic models in the literature, each seemingly resulting in different descriptions of viscoelastic constitutive behaviour and/or stressrelaxation and creep functions. Moreover, most studies do not apply a single discrete element model to characterise both stress-relaxation and creep behaviours of tissues. The underlying assumption for this disparity is the implicit perception that the viscoelasticity of soft tissues cannot be described by a universal behaviour or law, resulting in the lack of a unified approach in the literature based on discrete element representations. This paper derives the constitutive equation for different viscoelastic models applicable to soft tissues with two characteristic times. It demonstrates that all possible configurations exhibit a unified and universal behaviour, captured by a single constitutive relationship between stress, strain and time as: $\sigma+A \dot{\sigma}+B \ddot{\sigma}=P \dot{\varepsilon}+Q \ddot{\varepsilon}$ . The ensuing stress-relaxation $G(t)$ and creep $J(t)$ functions are also unified and universal, derived as $G(t)=c_{1} e^{\frac{-A+\sqrt{A^{2}-4 B}}{2 B} t}+\left(\sigma_{0}-c_{1}\right) e^{\frac{-A-\sqrt{A^{2}-4 B}}{2 B} t} \quad$ and $J(t)=c_{2}+\left(\varepsilon_{0}-c_{2}\right) e^{-\frac{P}{Q} t}+\frac{\sigma_{0}}{P} t$, respectively. Application of these relationships to experimental data is illustrated for various tissues including the aortic valve, ligament and cerebral artery. The unified model presented in this paper may be applied to all tissues with two characteristic times, obviating the need for employing varied configurations of discrete element models in preliminary investigation of the viscoelastic behaviour of soft tissues.

Keywords: viscoelasticity, discrete element models, stress-relaxation, creep, unified constitutive equation, soft tissues. 


\section{Unified viscoelasticity: \\ Applying discrete element models to soft tissues with two characteristic times}

\section{Introduction}

Biological tissues are known to exhibit complex constitutive behaviour, in which stress may depend on both strain and strain rate, as well as strain history (Fung, 1993; Bischoff et al., 2004). These characteristics suggest a behaviour that combines the properties of elastic solids and viscous fluids, and therefore biological tissues are generally known to respond in a viscoelastic manner to mechanical perturbations (Jamison et al., 1968). From a biomechanics point of view, the difference between the viscoelastic response of a tissue, and purely elastic or viscous responses, lies essentially in the relationship between stress, strain and time.

To address such relationships in soft tissues, appropriate models that describe a mathematical representation of the phenomenon of viscoelasticity are required. While some studies suggest that aspects of viscoelastic behaviour of tissues, such as stressrelaxation and creep, may be initiated at the extracellular-matrix level, e.g. by the viscous fluid-like behaviour and characteristics of glycosaminoglycans (GAGs) (Ratcliffe and Mow, 1996; Anssari-Benam et al., 2011a; Anssari-Benam et al., 2011b) and fibre-sliding (Gupta et al., 2010; Screen et al., 2013), rheological models have traditionally been the popular choice to characterise the viscoelastic behaviour and properties of soft tissues.

In general, rheological models correspond to either continuous spectral, or discrete element, mathematical representations (Jamison et al., 1968; Fung, 1993; Li and Xu, 2006). While the physical demonstration of both models is manifested by configurations of a finite number of springs and dashpots, the corresponding mathematical expressions are addressed differently. Continuous spectral representations are obtained by solving an integral equation for the relaxation spectrum, under the assumption that the applied strain and the resulting stress response are known (Jamison et al., 1968). Perhaps the most celebrated type of these models utilised in biomechanical studies is the quasi-linear viscoelasticity (QLV) 
model, successfully applied to various tissues ranging from cardiac muscles (Pinto and Patitucci, 1980) to ligaments (Woo et al., 1993) and tendons (Sarver et al., 2003). The governing equations for discrete element models are derived based on the local stress/strain/strain rate equations of the incorporated spring and dashpot elements, and how they are related to each other and with the global stress-strain relationships (Jamison et al., 1968). Examples of application of discrete element models to soft tissues include characterisation of the rate effects (Anssari-Benam et al., 2011a) and stress-relaxation and creep behaviour of heart valves (Liao et al, 2007; AnssariBenam et al., 2011b), tendons (Hooley and Cohen, 1979) and arteries (Rehal et al., 2006).

While the application of discrete element models to soft tissues have mainly been made within the context of linear viscoelasticity, which would undoubtedly introduce approximations and simplifications to the analysis (for example see Provenzano et al., 2002 and Anssari-Benam, 2014), discrete element models have been reported to provide good agreement with the experimental data. The preliminary experiments in characterising the viscoelasticity of soft tissues incorporate quasi-static loading regimes often in the form of stress-relaxation and creep tests, as well as tensile loading under various deformation rates. Discrete element viscoelastic models facilitate the quantification of the respective stress-relaxation and creep moduli, characteristic times, and the rate effects, in a mathematically and conceptually easy way, by incorporating combination of spring and dashpot elements. Additionally, different trends of experimental data may be fitted to these models relatively easily, by altering the number of the elements in the model or the model configuration. These attributes have made discrete element viscoelastic models a popular choice in investigating and characterising the viscoelasticity of soft tissues.

However, this apparent freedom in employing various element numbers or configurations has rendered a diverse array of viscoelastic models in the literature, each seemingly resulting in different descriptions of viscoelastic constitutive behaviour. This diversity, in turn, has given rise to a perception that the constitutive viscoelastic relationship between stress, strain and time in soft tissues may not be universal when characterised using discrete element models, but rather may depend on the employed number and configuration of the elements. Such disparity has made 
direct comparisons between the quantified viscoelastic properties of different tissues highly problematic, as the differences in values may be partly attributed to the application of 'different' viscoelastic models. This problem becomes more pronounced when comparing the reported viscoelastic properties of a specific soft tissue, where different configurations have been employed. For example, different relaxation times for aortic valve tissue have been reported when characterised by QLV (Sauren et al., 1983) which is a Kelvin-based model, Maxwell-type exponential decay (Lee and Vesely, 1995) and Prony series (Anssari-Benam et al., 2011b). A similar diversity of parametric values has been identified in the literature for arteries, tendons and other soft tissues.

Due care must therefore be observed in choosing adequate number and appropriate configuration of elements that could suitably describe the experimentally observed viscoelastic behaviour of soft tissues. Previous studies have established that two characteristic time scales, referred to as "fast" and "slow" times or "short-" and "long-" time memory, are sufficient to capture and characterise the time-dependent behaviour of many soft tissues (Fung, 1993; Pioletti and Rakotomanana, 2000; Banks et al., 2011). The fast and slow characteristic times are the macroscopic time scales that are required for a tissue to return to its equilibrium state, after exposure to external mechanical perturbations. The fast characteristic time implies that a short time is required for the tissue to retain the equilibrium state, while a slow characteristic time reflects a long time-scale for the tissue to return to its original reference. Soft tissues possess both short and long characteristic time scales, presenting a fast initial recovery followed by a much slower equilibrium kinematics, in stress-relaxation tests. In discrete element modelling, this two-characteristic time behaviour can be represented by two dashpots, the mechanical elements introducing the time/rate effects, together with two spring elements, in an arrangement such that similar elements would not form parallel or series configurations. In addition, most soft tissues exhibit both stress-relaxation and creep behaviours when subjected to the respective loading conditions. An appropriate viscoelastic model therefore must also be capable of characterising both those behaviours under those conditions.

These critical axioms, however, have often been overlooked in the discrete element models developed in the literature. Indeed, literature suggests a variety of Kelvin- 
based, Maxwell-based, or standard linear solid type discrete element models that have been used for different tissues, or even for the same tissue, with various element numbers, only suitable for characterising a particular viscoelastic behaviour. For example, the standard linear solid model while successful in addressing forcedisplacement relationships in some biological entities (e.g. axonal microtubules by Shamloo et al., 2015), can only render a single characteristic relaxation time and as such may not be applicable to tissues with two characteristic times. Similarly, Maxwell-based models can only accurately characterise stress-relaxation, and Kelvinbased models can only accurately characterise creep behaviour of soft tissues. Studies have therefore often favoured the application of one model to describe the stressrelaxation and a separate model to describe the creep behaviour, even for the same tissue (Hooley and Cohen, 1979; Thornton et al. 1997; Anssari-Benam et al., 2011b). The biomechanics literature has therefore not adequately addressed the important question of whether the viscoelasticity of soft tissues with two characteristic times is a universal behaviour, and has subsequently not provided a unified discrete element model with single constitutive relationship between stress, strain and time, or stress relaxation and creep functions applicable to all such tissues.

In this paper, the constitutive equation for discrete element viscoelastic models applicable to soft tissues with two characteristic times, i.e. four-element representations, are derived and presented. It is shown that all possible configurations of these models exhibit a universal behaviour, with the following unified relationship between stress, strain and time:

$$
\left\{\begin{array}{l}
\sigma+A \dot{\sigma}+B \ddot{\sigma}=P \dot{\varepsilon}+Q \ddot{\varepsilon} \\
G(t)=c_{1} e^{\frac{-A+\sqrt{A^{2}-4 B}}{2 B} t}+\left(\sigma_{0}-c_{1}\right) e^{\frac{-A-\sqrt{A^{2}-4 B}}{2 B} t} \\
J(t)=c_{2}+\left(\varepsilon_{0}-c_{2}\right) e^{-\frac{P}{Q} t}+\frac{\sigma_{0}}{P} t
\end{array}\right.
$$

where $\sigma$ denotes stress, $\varepsilon$ is strain, $G(t)$ and $J(t)$ are the resulting stress relaxation and creep functions, respectively, and $A, B, P$ and $Q$ are constants determined from elastic and viscous damping moduli of the elements in the model, as will be shown in the next section. Our analysis concludes that different four-element viscoelastic 
models applicable to soft tissues with two characteristic times, all lead to a universal response, characterised by a single mathematical representation given in equation (1), with single universal stress-relaxation and creep functions. Application of these functions to experimental data obtained from stress-relaxation and creep tests reported in the literature for a range of different types of tissues including aortic valve, ligament and cerebral artery is also presented.

\section{Independent discrete element viscoelastic models with two characteristic times}

The possible five independent discrete element viscoelastic models containing two dashpots and springs, and exhibiting two characteristic times, are shown in Figure 1. All other combinations of springs and dashpots may either not result in two characteristic times, or may form elements in parallel or series which may reduce to one of the five considered discrete model arrangements presented. It can be shown how the unified viscoelastic relationship in equation (1a) mathematically describes the constitutive behaviour between stress, strain and time for all of these models, characterising a universal viscoelastic behaviour.

In general, the following steps may be applied to each model to derive the unified equation (equation (1a)) from the local stress/strain relationships. An 'element' is defined as a basic spring or dashpot component, while a 'segment' is defined as a combination of those elements either in parallel or series. Accordingly, the local relationships between the stress/strain of the elements and/or the segments are hence expressed. Then the first and second derivatives of those stress/strain relationships are obtained, and are combined together to formulate a second-order differential equation between the global stress and strain. The coefficients of the derivatives are adjusted to retrieve the unified relationship given in equation (1a).

The above described approach is detailed in the following for the model shown in Figure 1a. In this model: 


$$
\left\{\begin{array}{l}
\sigma=\sigma_{D 1} \\
\sigma_{D 1}=\sigma_{S 1}+\sigma_{S 2} \\
\sigma_{D 2}=\sigma_{S 2} \\
\varepsilon=\varepsilon_{D 1}+\varepsilon_{D 2}+\varepsilon_{S 2} \\
\varepsilon_{S 1}=\varepsilon_{D 2}+\varepsilon_{S 2}
\end{array}\right.
$$

Note that subscripts $S_{S}$ and ${ }_{D}$ denote the parameter of interest related to spring and dashpot elements, respectively.

Differentiating equation (2e) with respect to time, and noting that equation (2c) describes $\eta_{2} \dot{\varepsilon}_{D 2}=E_{2} \varepsilon_{S 2}$ results in:

$$
\dot{\varepsilon}_{S 1}=\frac{E_{2}}{\eta_{2}} \varepsilon_{S 2}+\dot{\varepsilon}_{S 2}
$$

Combining equations (2a) and (2b):

$$
\sigma=E_{1} \varepsilon_{S 1}+E_{2} \varepsilon_{S 2}
$$

Taking the time derivative of equation (4) and dividing both sides by $\eta_{2}$ :

$$
\frac{\dot{\sigma}}{\eta_{2}}=\frac{E_{1}}{\eta_{2}} \dot{\varepsilon}_{S 1}+\frac{E_{2}}{\eta_{2}} \dot{\varepsilon}_{S 2}
$$

Differentiating equation (4) twice with respect to time and dividing both sides by $E_{2}$ :

$$
\frac{\ddot{\sigma}}{E_{2}}=\frac{E_{1}}{E_{2}} \ddot{\varepsilon}_{S 1}+\ddot{\varepsilon}_{S 2}
$$

Summing equations (5) and (6):

$$
\frac{\dot{\sigma}}{\eta_{2}}+\frac{\ddot{\sigma}}{E_{2}}=\frac{E_{1}}{\eta_{2}} \dot{\varepsilon}_{S 1}+\frac{E_{2}}{\eta_{2}} \dot{\varepsilon}_{S 2}+\frac{E_{1}}{E_{2}} \ddot{\varepsilon}_{S 1}+\ddot{\varepsilon}_{S 2}
$$

Now, differentiating equation (3) with respect to time:

$$
\frac{E_{2}}{\eta_{2}} \dot{\varepsilon}_{S 2}=\ddot{\varepsilon}_{S 1}-\ddot{\varepsilon}_{S 2}
$$

Substituting equation (8) into (7):

$$
\frac{\dot{\sigma}}{\eta_{2}}+\frac{\ddot{\sigma}}{E_{2}}=\frac{E_{1}}{\eta_{2}} \dot{\varepsilon}_{S 1}+\left(1+\frac{E_{1}}{E_{2}}\right) \ddot{\varepsilon}_{S 1}
$$


From equations (2a), (2d) and (2e):

$$
\sigma=\eta_{1} \dot{\varepsilon}_{D 1}=\eta_{1}\left(\dot{\varepsilon}-\dot{\varepsilon}_{S 1}\right) \Rightarrow\left\{\begin{array}{l}
\dot{\varepsilon}_{S 1}=\dot{\varepsilon}-\frac{\sigma}{\eta_{1}} \\
\ddot{\varepsilon}_{S 1}=\ddot{\varepsilon}-\frac{\dot{\sigma}}{\eta_{1}}
\end{array}\right.
$$

Substituting expressions for $\dot{\varepsilon}_{S 1}$ and $\ddot{\varepsilon}_{S 1}$ provided in equation (10) into equation (9):

$$
\frac{E_{1}}{\eta_{1} \eta_{2}} \sigma+\left(\frac{1}{\eta_{1}}+\frac{1}{\eta_{2}}+\frac{E_{1}}{E_{2} \eta_{1}}\right) \dot{\sigma}+\frac{\ddot{\sigma}}{E_{2}}=\frac{E_{1}}{\eta_{2}} \dot{\varepsilon}+\left(1+\frac{E_{1}}{E_{2}}\right) \ddot{\varepsilon}
$$

which can be re-arranged as:

$$
\sigma+\left(\frac{\eta_{1}+\eta_{2}}{E_{1}}+\frac{\eta_{2}}{E_{2}}\right) \dot{\sigma}+\frac{\eta_{1} \eta_{2}}{E_{1} E_{2}} \ddot{\sigma}=\eta_{1} \dot{\varepsilon}+\frac{\eta_{1} \eta_{2}}{E_{1}}\left(1+\frac{E_{1}}{E_{2}}\right) \ddot{\varepsilon}
$$

Equation (12) is the constitutive relationship between stress, strain and time for this viscoelastic model. It immediately follows that equation (12) is reminiscent of equation (1a), with $A=\left(\frac{\eta_{1}+\eta_{2}}{E_{1}}+\frac{\eta_{2}}{E_{2}}\right), B=\frac{\eta_{1} \eta_{2}}{E_{1} E_{2}}, P=\eta_{1}$ and $Q=\frac{\eta_{1} \eta_{2}}{E_{1}}\left(1+\frac{E_{1}}{E_{2}}\right)$.

The constitutive equation between stress, strain and time for the remaining four model representations shown in Figures (1b) to (1e) is also governed by the universal relationship given in equation (1a), derived and presented in Appendix A for the interested reader.

It must be noted that a model consisting of two Kelvin elements in series, as shown in Figure 2, would not represent a relaxation behaviour with two characteristic decay times, as the governing equation of this model is: $\sigma+A \dot{\sigma}=R \varepsilon+P \dot{\varepsilon}+Q \ddot{\varepsilon}$. Thus, this model may not be deemed suitable for application to soft tissues with two characteristic times, and has therefore not been included in our analysis.

\section{Universal stress-relaxation and creep functions}

Both stress-relaxation and creep functions are derived from the universal constitutive relationship between stress, strain and time governing all of the above 
models, $\sigma+A \dot{\sigma}+B \ddot{\sigma}=P \dot{\varepsilon}+Q \ddot{\varepsilon}$, by applying the appropriate boundary conditions. The phenomenon of stress relaxation incurs while the strain is kept constant, therefore $\dot{\varepsilon}=\ddot{\varepsilon}=0 \Rightarrow \sigma+A \dot{\sigma}+B \ddot{\sigma}=0$. Solving this second order differential equation for stress gives the relaxation function $G(t)$ as:

$$
G(t)=c_{1} e^{r_{1} t}+\left(\sigma_{0}-c_{1}\right) e^{r_{2} t}
$$

where $c_{1}$ is a constant, $\sigma_{0}$ is the initial stress prior to relaxation, $r_{1}$ and $r_{2}$ are: $r_{1,2}=\frac{-A \pm \sqrt{A^{2}-4 B}}{2 B}$, and are related to relaxation times as: $\tau_{1,2}=-\frac{1}{r_{1,2}}$.

Creep occurs while stress is kept constant at an arbitrary level $\sigma_{0}$, therefore $\dot{\sigma}=\ddot{\sigma}=0 \Rightarrow P \dot{\varepsilon}+Q \ddot{\varepsilon}=\sigma_{0}$. Solving this equation for strain results in the creep function $J(t)$ in the form:

$$
J(t)=c_{2}+\left(\varepsilon_{0}-c_{2}\right) e^{r t}+\frac{\sigma_{0}}{P} t
$$

where $c_{2}$ is a constant, $\varepsilon_{0}$ is the initial strain prior to creep, $r$ is: $r=-\frac{P}{Q}$, and is related to retardation time as: $\tau=-\frac{1}{r}$.

Equations (13) and (14) describe the universal stress-relaxation and creep behaviour of viscoelastic tissues with two characteristic times. It is worth noting that the unified constitutive viscoelastic behaviour accommodates both stress-relaxation and creep, precluding the need to adopt separate models to characterise each behaviour.

In order to corroborate the application of these functions in characterising the timedependent behaviour of soft tissues, $G(t)$ and $J(t)$ were fitted to the experimental stress-relaxation and creep data of a range of soft tissue specimens including aortic valve, ligament and cerebral artery, reported in the literature. The experimental details of sample preparation and test protocols have been described in the respective studies. In brief, sample specimens of porcine aortic valve (Anssari-Benam et al., 2011b), rabbit medial collateral ligament (Thornton et al., 1997; Thornton et al., 
2001) and human middle cerebral artery ( $\mathrm{Li}$ et al., 2013) were subjected to uniaxial stress relaxation and creep tests using universal mechanical testing machines, over the periods ranging from 5 minutes to 2 hours. Representative examples were considered for stress relaxation at constant strain levels of $6 \%$ of the failure strain, $5 \%$ and $25.2 \%$ for the aortic valve, ligament and cerebral artery specimens, respectively. Representative creep samples were selected at constant stress levels of $5 \%$ of the failure stress, $14 \mathrm{MPa}$ and $18.7 \mathrm{kPa}$ for the aortic valve, ligament and cerebral artery specimens, respectively. This range was chosen to accommodate the viscoelastic behaviour of a diverse range of soft tissues under both low and high levels of stress/strain, over an inclusive period of time.

Figure 3 shows how $G(t)$ and $J(t)$ functions described the respective stressrelaxation and creep behaviours. The fitting procedure was performed by Curve Fitting Toolbox ${ }^{\mathrm{TM}}$ in MATLAB ${ }^{\circledR}$, using Levenberg-Marquardt algorithm, reporting $R^{2}$ values in excess of 0.98. Fitting parameters are listed in Table 1. The results highlight the derived unified model provides a very good fit to the experiments data, capturing both stress-relaxation and creep behaviours of the subject tissues.

\section{Concluding remarks}

The time-dependent behaviour of soft tissues can be characterised by two characteristic time scales, known as 'fast' and 'slow' times, or 'short-' and 'long-' time memory scales. Characterising this behaviour incorporates two dashpots, together with two spring elements, in discrete element representation models of viscoelasticity. A unified and universal relationship between stress, strain and time, governing the constitutive behaviour of such models was derived and presented. Our analysis indicates that the universal viscoelastic behaviour of tissues with two characteristic times is independent of the chosen configurations and arrangements of spring and dashpot elements. This may therefore obviate the need to employ different models to characterise the viscoelastic behaviour of different soft tissues.

Using this constitutive relationship (equation (1a)), stress-relaxation and creep functions were derived and presented (equations (13 and (14)). The universal 
viscoelastic behaviour of four-element discrete models is therefore capable of capturing and characterising both stress-relaxation and creep. To corroborate the application of these functions to experimental data, the derived model was fitted to data collated from stress-relaxation and creep tests of aortic valve, ligament and cerebral artery specimens, showing an acceptable agreement between the model and experiment. It must be noted that other experimental procedures such as dynamic mechanical tests may also be used in characterising viscoelastic behaviour of tissues. However, those tests are most useful in determining the storage and loss moduli, and the phase lag between stress and strain in frequency domains. Such analysis may therefore not be directly relevant to characterising the stress relaxation and creep behaviours of soft tissues, or the characteristic times, and was not considered in this study.

One of the main advantages of deriving this unified constitutive equation is that it will allow comparisons of the characterised viscoelastic parameters between different tissues, and facilitate establishing universal values of viscoelastic parameters for a soft tissue. Model parameters $A, B, P$ and $Q$ can be quantified from relevant experimental data and be directly compared between different tissues. Furthermore, relationships between model constants $\left(E_{i}\right.$ and $\left.\eta_{i}\right)$ and material parameters, such as relaxation times, may be established. Thus, differences between the values of material parameters of different tissues can now be interpreted based on the differences in known model constants. In view of the present analysis, the application of the presented model in this paper is recommended in preliminary investigations of the time-dependent behaviour of soft tissues with two-characteristic times. 


\section{Appendix A}

For the model in Figure 1b:

$$
\left\{\begin{array}{l}
\sigma=\sigma_{S 1}+\sigma_{D 1}=\sigma_{S 2}=\sigma_{D 2} \\
\varepsilon=\varepsilon_{S 1}+\varepsilon_{S 2}+\varepsilon_{D 2} \\
\varepsilon_{S 1}=\varepsilon_{D 1}
\end{array}\right.
$$

From equation (A1a) it follows that:

$$
\left\{\begin{array}{l}
\sigma=E_{2} \varepsilon_{S 2} \\
\sigma=E_{1} \varepsilon_{S 1}+\eta_{1} \dot{\varepsilon}_{D 1} \\
\sigma=\eta_{2} \dot{\varepsilon}_{D 2}
\end{array}\right.
$$

Differentiating equation (A1b) with respect to time, and using (A2a) and (A2c):

$$
\dot{\varepsilon}=\dot{\varepsilon}_{S 1}+\frac{\dot{\sigma}}{E_{2}}+\frac{\sigma}{\eta_{2}}
$$

Differentiating the above equation with respect to time:

$$
\ddot{\varepsilon}=\ddot{\varepsilon}_{S 1}+\frac{\ddot{\sigma}}{E_{2}}+\frac{\dot{\sigma}}{\eta_{2}}
$$

Multiplying equation (A3) by $E_{1}$ and equation (A4) by $\eta_{1}$, and adding the resulting relationships:

$$
E_{1} \dot{\varepsilon}+\eta_{1} \ddot{\varepsilon}=E_{1} \dot{\varepsilon}_{S 1}+\eta_{1} \ddot{\varepsilon}_{S 1}+\frac{E_{1}}{E_{2}} \dot{\sigma}+\frac{\eta_{1}}{E_{2}} \ddot{\sigma}+\frac{E_{1}}{\eta_{2}} \sigma+\frac{\eta_{1}}{\eta_{2}} \dot{\sigma}
$$

Now, taking the time derivative from equation (A2b) and re-arranging it:

$$
E_{1} \dot{\varepsilon}_{S 1}=\dot{\sigma}-\eta_{1} \ddot{\varepsilon}_{D 1}
$$

Substituting equation (A6) into (A5):

$$
\frac{E_{1}}{\eta_{2}} \sigma+\left(1+\frac{E_{1}}{E_{2}}+\frac{\eta_{1}}{\eta_{2}}\right) \dot{\sigma}+\frac{\eta_{1}}{E_{2}} \ddot{\sigma}=E_{1} \dot{\varepsilon}+\eta_{1} \ddot{\varepsilon}
$$

which can be re-written as: 


$$
\sigma+\left(\frac{\eta_{1}+\eta_{2}}{E_{1}}+\frac{\eta_{2}}{E_{2}}\right) \dot{\sigma}+\frac{\eta_{1} \eta_{2}}{E_{1} E_{2}} \ddot{\sigma}=\eta_{2} \dot{\varepsilon}+\frac{\eta_{1} \eta_{2}}{E_{1}} \ddot{\varepsilon}
$$

Equation (A8) is the constitutive relationship between stress, strain and time for this model. It is evident that this equation has the same form as equation (1a), with $A=\left(\frac{\eta_{1}+\eta_{2}}{E_{1}}+\frac{\eta_{2}}{E_{2}}\right), B=\frac{\eta_{1} \eta_{2}}{E_{1} E_{2}}, P=\eta_{2}$ and $Q=\frac{\eta_{1} \eta_{2}}{E_{1}}$

For the model shown in Figure 1c:

$$
\left\{\begin{array}{l}
\sigma=\sigma_{D 1}=\sigma_{S 1} \\
\sigma=\sigma_{S 2}+\sigma_{D 2} \\
\varepsilon=\varepsilon_{D 1}+\varepsilon_{D 2}+\varepsilon_{S 1} \\
\varepsilon_{D 2}=\varepsilon_{S 2}
\end{array}\right.
$$

Equation (A9) is similar to the governing equation of model shown in Figure 1b (see equation (A1)). Using the same steps described in §2, i.e. differentiating equation (A9c) with respect to time and multiplying it by $E_{2}$; multiplying the second time derivative of equation (A9c) by $\eta_{2}$, and adding the resulting two equations, one arrives at:

$$
\sigma+\left(\frac{\eta_{1}+\eta_{2}}{E_{2}}+\frac{\eta_{1}}{E_{1}}\right) \dot{\sigma}+\frac{\eta_{1} \eta_{2}}{E_{1} E_{2}} \ddot{\sigma}=\eta_{1} \dot{\varepsilon}+\frac{\eta_{1} \eta_{2}}{E_{2}} \ddot{\varepsilon}
$$

Equation (A10) is the constitutive equation describing the relationship between stress, strain and time for this model. This equation is the same as equation (1a), with $A=\left(\frac{\eta_{1}+\eta_{2}}{E_{2}}+\frac{\eta_{1}}{E_{1}}\right), B=\frac{\eta_{1} \eta_{2}}{E_{1} E_{2}}, P=\eta_{1}$ and $Q=\frac{\eta_{1} \eta_{2}}{E_{2}}$.

For the model presented in Figure 1d: 


$$
\left\{\begin{array}{l}
\sigma=\sigma_{S 1} \\
\sigma_{S 1}=\sigma_{D 1}+\sigma_{D 2} \\
\sigma_{S 2}=\sigma_{D 2} \\
\varepsilon=\varepsilon_{S 1}+\varepsilon_{D 2}+\varepsilon_{S 2} \\
\varepsilon_{D 1}=\varepsilon_{D 2}+\varepsilon_{S 2}
\end{array}\right.
$$

One can note that equation (A11) is similar in format to the governing equations of the model presented in Figure 1a, described by equation (2). Using the same approach, i.e. eliminating the local strains and their corresponding stresses by taking the first and second time derivative of equation (A11a) by substituting (A11b) and (A11c), multiplying each with $\eta_{1}$ and $E_{1}$, and adding the resulting equations one arrives at:

$$
\sigma+\left(\frac{\eta_{1}+\eta_{2}}{E_{1}}+\frac{\eta_{2}}{E_{2}}\right) \dot{\sigma}+\frac{\eta_{1} \eta_{2}}{E_{1} E_{2}} \ddot{\sigma}=\left(\eta_{1}+\eta_{2}\right) \dot{\varepsilon}+\frac{\eta_{1} \eta_{2}}{E_{1}} \ddot{\varepsilon}
$$

Equation (A12) is the constitutive equation of this model, and is similar to equation (1a) with $A=\left(\frac{\eta_{1}+\eta_{2}}{E_{1}}+\frac{\eta_{2}}{E_{2}}\right), B=\frac{\eta_{1} \eta_{2}}{E_{1} E_{2}}, P=\eta_{1}+\eta_{2}$ and $Q=\frac{\eta_{1} \eta_{2}}{E_{1}}$.

Finally, for the model shown in Figure 1e which constitutes of two Maxwell elements in parallel:

$$
\left\{\begin{array}{l}
\sigma=\sigma_{S 1}+\sigma_{S 2} \\
\sigma_{S 1}=\sigma_{D 1} \\
\sigma_{S 2}=\sigma_{D 2} \\
\varepsilon=\varepsilon_{S 1}+\varepsilon_{D 1} \\
\varepsilon_{S 1}+\varepsilon_{D 1}=\varepsilon_{S 2}+\varepsilon_{D 2}
\end{array}\right.
$$


From equations (A13d) and (A13e) it follows that:

$$
\left\{\begin{array} { l } 
{ \varepsilon = \varepsilon _ { S 1 } + \varepsilon _ { D 1 } } \\
{ \varepsilon = \varepsilon _ { S 2 } + \varepsilon _ { D 2 } }
\end{array} \Rightarrow \left\{\begin{array}{l}
\dot{\varepsilon}=\dot{\varepsilon}_{S 1}+\dot{\varepsilon}_{D 1} \\
\dot{\varepsilon}=\dot{\varepsilon}_{S 2}+\dot{\varepsilon}_{D 2}
\end{array}\right.\right.
$$

and from equations (A13b) and (A13c):

$$
\left\{\begin{array} { l } 
{ E _ { 1 } \varepsilon _ { S 1 } = \eta _ { 1 } \dot { \varepsilon } _ { D 1 } } \\
{ E _ { 2 } \varepsilon _ { S 2 } = \eta _ { 2 } \dot { \varepsilon } _ { D 2 } }
\end{array} \Rightarrow \left\{\begin{array}{l}
\dot{\varepsilon}_{D 1}=\frac{E_{1}}{\eta_{1}} \varepsilon_{S 1} \\
\dot{\varepsilon}_{D 2}=\frac{E_{2}}{\eta_{2}} \varepsilon_{S 2}
\end{array}\right.\right.
$$

Substituting equation (A15) into (A14):

$$
\left\{\begin{array}{l}
\dot{\varepsilon}=\frac{E_{1}}{\eta_{1}} \varepsilon_{S 1}+\dot{\varepsilon}_{S 1} \\
\dot{\varepsilon}=\frac{E_{2}}{\eta_{2}} \varepsilon_{S 2}+\dot{\varepsilon}_{S 2}
\end{array}\right.
$$

From equation (A13a):

$$
\sigma=E_{1} \varepsilon_{S 1}+E_{2} \varepsilon_{S 2} \Rightarrow \varepsilon_{S 1}=\frac{\sigma-E_{2} \varepsilon_{S 2}}{E_{1}}
$$

Substituting for $\varepsilon_{S 1}$ from equation (A17) into (A16a):

$$
\dot{\varepsilon}_{S 2}=\frac{E_{1}}{E_{2} \eta_{1}} \sigma-\frac{E_{1}}{\eta_{1}} \varepsilon_{S 2}+\frac{\dot{\sigma}}{E_{2}}-\frac{E_{1}}{E_{2}} \dot{\varepsilon}
$$

and substituting equation (A18) into (A16b):

$$
\varepsilon_{S 2}=\frac{\eta_{1} \eta_{2}\left(E_{1}+E_{2}\right)}{E_{2}\left(\eta_{1} E_{2}-\eta_{2} E\right)_{1}} \dot{\varepsilon}+\frac{E_{1} \eta_{2}}{E_{2}\left(\eta_{2} E_{1}-\eta_{1} E_{2}\right)} \sigma+\frac{\eta_{1} \eta_{2}}{E_{2}\left(\eta_{2} E_{1}-\eta_{1} E_{2}\right)} \dot{\sigma}
$$

Now, rearranging equation (A18) for $\varepsilon_{S 2}$ :

$$
\varepsilon_{S 2}=-\frac{\eta_{1}}{E_{1}} \dot{\varepsilon}_{S 2}+\frac{1}{E_{2}} \sigma+\frac{\eta_{1}}{E_{1} E_{2}} \dot{\sigma}-\frac{\eta_{1}}{E_{2}} \dot{\varepsilon}
$$

Substituting equation (A20) into (A16b):

$$
\dot{\varepsilon}_{S 2}=\frac{E_{1} \eta_{2}+E_{1} \eta_{1}}{E_{1} \eta_{2}-\eta_{1} E_{2}} \dot{\varepsilon}-\frac{E_{1}}{E_{1} \eta_{2}-\eta_{1} E_{2}} \sigma-\frac{\eta_{1}}{E_{1} \eta_{2}-\eta_{1} E_{2}} \dot{\sigma}
$$


Differentiating equation (A19) with respect to time, and equating it with equation (A21), results in:

$$
\sigma+\left(\frac{E_{1} \eta_{2}+E_{2} \eta_{1}}{E_{1} E_{2}}\right) \dot{\sigma}+\frac{\eta_{1} \eta_{2}}{E_{1} E_{2}} \ddot{\sigma}=\left(\eta_{1}+\eta_{2}\right) \dot{\varepsilon}+\left(\frac{E_{1} \eta_{1} \eta_{2}+E_{2} \eta_{1} \eta_{2}}{E_{1} E_{2}}\right) \ddot{\varepsilon}
$$

Equation (A22) is the constitutive equation for this viscoelastic model (Figure 1e), describing the relationship between stress, strain and time in this model. It immediately follows that equation (A22) is the same as equation (1a), with $A=\left(\frac{E_{1} \eta_{2}+E_{2} \eta_{1}}{E_{1} E_{2}}\right), B=\frac{\eta_{1} \eta_{2}}{E_{1} E_{2}}, P=\eta_{1}+\eta_{2}$ and $Q=\left(\frac{E_{1} \eta_{1} \eta_{2}+E_{2} \eta_{1} \eta_{2}}{E_{1} E_{2}}\right)$. 


\section{References}

Anssari-Benam, A., Bader, D. L., Screen, H. R. C., 2011a. A combined experimental and modelling approach to aortic valve viscoelasticity in tensile deformation. Journal of Materials Science: Materials in Medicine 22 (2), 253-262.

Anssari-Benam, A., Bader, D. L., Screen, H. R. C., 2011b. Anisotropic timedependant behaviour of the aortic valve. Journal of the Mechanical Behavior of Biomedical Materials 4 (8), 1603-1610.

Anssari-Benam, A., 2014. Is the time-dependent behaviour of the aortic valve intrinsically quasi-linear? Mechanics of Time-Dependent Materials 18 (2), 339-348.

Banks, H. T., Hu, S., Kenz, Z. R., 2011. A brief review of elasticity and viscoelasticity for solids. Advances in Applied Mathematics and Mechanics 3 (1), 151.

Bischoff, J. E., Arruda, E. M., Grosh, K., 2004. A rheological network model for the continuum anisotropic and viscoelastic behaviour of soft tissue. Biomechanics and Modeling in Mechanobiology 3 (1), 56-65.

Fung, Y. C., 1993. Biomechanics: Mechanical properties of living tissue. $2^{\text {nd }}$ edition, Springer-Verlag New York Inc., New York.

Gupta, H. S., Seto, J., Krauss, S., Boesecke, P., Screen, H. R. C., 2010. In situ multilevel analysis of viscoelastic deformation mechanisms in tendon collagen. Journal of Structural Biology 169 (2), 183-191.

Hooley, C. J., Cohen, R. E., 1979. A model for the creep behaviour of tendon. International Journal of Biological Macromolecules 1 (3), 123-132.

Jamison, C. E., Marangoni, R. D., Glaser, A. A., 1968. Viscoelastic properties of soft tissue by discrete model characterization. Journal of Biomechanics 1 (1), 33-46.

Lee, M. K., Vesely, I., 1995. An investigation of the stress relaxation spectrum of the aortic valve, In Proceedings of the $17^{\text {th }}$ IEEE Annual Conference, Canada, Montreal.

Li, Y., Xu, M., 2006. Hysteresis and precondition of viscoelastic solid models. Mechanics of Time-Dependent Materials 10 (2), 113-123.

Li, D., Xu, D., Li, P., Wei, J., Yang, K., Zhao, C., 2013. Viscoelastic evaluation of fetal umbilical vein for reconstruction of middle cerebral artery. Neural Regeneration Research 8 (32), 3055-3062.

Liao, J., Yang, L., Grashow, J., Sacks, M. S., 2007. The relation between collagen fibril kinematics and mechanical properties in the mitral valve anterior leaflet. Journal of Biomechanical Engineering 129 (1), 78-87. 
Pinto, J. G., Patitucci, P. J., 1980. Visco-elasticity of passive cardiac muscle. Journal of Biomechanical Engineering 102 (1), 57-61.

Pioletti, D. P., Rakotomanana, L. R., 2000. Non-linear viscoelastic laws for soft biological tissues. European Journal of Mechanics - A/Solids 19 (5), 749-759.

Provenzano, P. P., Lakes, R. S., Corr, D. T., Vanderby Jr., R., 2002. Application of nonlinear viscoelastic models to describe ligament behaviour. Biomechanics and Modeling in Mechanobiology 1 (1), 45-57.

Ratcliffe, A., Mow, V. C., 1996. Articular cartilage. In: Comper, W. D. (Ed), Extracellular Matrix, vol. 1. Harwood Academic Publishers, Amsterdam, pp. 268-272.

Rehal, D., Guo, X., Lu, X., Kassab, G. S., 2006. Duration of no-load state affects opening angle of porcine coronary arteries. American Journal of Physiology - Heart and Circulatory Physiology 290 (5), H1871-H1878.

Sarver, J. J., Robinson, P. S., Elliott, D. M., 2003. Methods for quasi-linear viscoelastic modelling of soft tissue: application to incremental stress-relaxation experiments. Journal of Biomechanical Engineering 125 (5), 754-758.

Sauren, A. A. H. J., van Hout, M. C., van Steenhoven, A. A., Veldpaus, F. E., Janssen, J. D., 1983. The mechanical properties of porcine aortic valve tissues. Journal of Biomechanics 16 (5), 327-337.

Screen, H. R. C., Toorani, S., Shelton, J. C., 2013. Microstructural stress relaxation mechanics in functionally different tendons. Medical Engineering \& Physics 35 (1), 96-102.

Shamloo, A., Manuchehrfar, F., Rafi-Tabar, H., 2015. A viscoelastic model for axonal microtubule rupture. Journal of Biomechanics 48 (7), 1241-1247.

Thornton, G. M., Frank, C. B., Shrive, N. G., 2001. Ligament creep behavior can be predicted from stress relaxation by incorporating fiber recruitment. Journal of Rheology 45 (2), 493-507.

Thornton, G. M., Oliynyk, A., Frank, C. B., Shrive, N. G., 1997. Ligament creep cannot be predicted from stress relaxation at low stress: a biomechanical study of the rabbit medial collateral ligament. Journal of Orthopaedic Research 15 (5), 652-656.

Woo, S. L. Y., Johnson, G. A., Smith, B. A., 1993. Mathematical modelling of ligaments and tendons. Journal of Biomechanical Engineering 115 (4B), 468-473. 


\section{Table legend}

Table 1- Stress-relaxation and creep parameters characterised by fitting $G(t)$ and $J(t)$ functions to the experimental data (Figure 3). Numerical values are presented as Mean \pm SD. 
Table 1

\begin{tabular}{|c|c|c|c|}
\hline \multicolumn{4}{|c|}{ Stress relaxation } \\
\hline & $c_{1}[\mathrm{MPa}]$ & $r_{1}\left[\mathrm{~s}^{-1}\right]$ & $r_{2}\left[\mathrm{~s}^{-1}\right]$ \\
\hline Porcine aortic valve & $0.0188 \pm 0.0004$ & $-0.0884 \pm 0.0008$ & $-2.44 \times 10^{-4} \pm 1.03 \times 10^{-5}$ \\
\hline Rabbit medial collateral ligament & $3.1840 \pm 0.1410$ & $-0.0250 \pm 0.0040$ & $-1.57 \times 10^{-4} \pm 6.96 \times 10^{-5}$ \\
\hline Human middle cerebral artery & $0.0030 \pm 0.00006$ & $0.0031 \pm 0.00008$ & $-6.59 \times 10^{-4} \pm 1.36 \times 10^{-6}$ \\
\hline \multicolumn{4}{|c|}{ Creep } \\
\hline & $C_{2}[-]$ & $r\left[\mathrm{~s}^{-1}\right]$ & $P[\mathrm{MPa} \mathrm{s}]$ \\
\hline Porcine aortic valve & $0.0825 \pm 0.0001$ & $-0.0440 \pm 0.0200$ & $4.40 \times 10^{4} \pm 8.32 \times 10^{3}$ \\
\hline Rabbit medial collateral ligament & $0.0550 \pm 0.0004$ & $-0.0245 \pm 0.0192$ & $4.90 \times 10^{6} \pm 9.70 \times 10^{5}$ \\
\hline Human middle cerebral artery & $0.2664 \pm 0.0007$ & $-0.0019 \pm 0.0009$ & $2.47 \times 10^{4} \pm 4.51 \times 10^{3}$ \\
\hline
\end{tabular}




\section{Figure legends}

Figure 1- Discrete element representation of independent viscoelastic models with two characteristic times, constraining two dashpots and springs.

Figure 2- Discrete element representation of a viscoelastic model consisting of two Kelvin elements in series.

Figure 3- Experimental data versus functions in equations (13) and (14) for stressrelaxation and creep behaviour of representative aortic valve, ligament and cerebral artery. Stress relaxation at: (a) $\varepsilon=6 \%$ of the failure strain of porcine aortic valve in circumferential direction; (b) $\varepsilon=5 \%$ of the rabbit medial collateral ligament; and (c) $\varepsilon=25.2 \%$ for human middle cerebral artery. Creep at: (d) $\sigma=5 \%$ of the failure stress of porcine aortic valve in circumferential direction; (e) $\sigma=14 \mathrm{MPa}$ for rabbit medial collateral ligament; and (f) $\sigma=18.7 \mathrm{kPa}$ for human middle cerebral artery. Hollow circles represent the experimental data, and continuous lines show the model trends. Experimental data were collated from Anssari-Benam et al. (2011b) for aortic valve, Thornton et al. (1997) and Thornton et al. (2001) for rabbit medial collateral ligament, and Li et al. (2013) for human middle cerebral artery. 
Figure 1

(a)

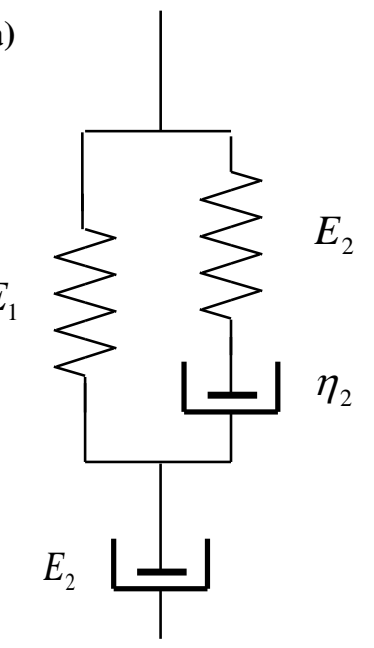

(d)

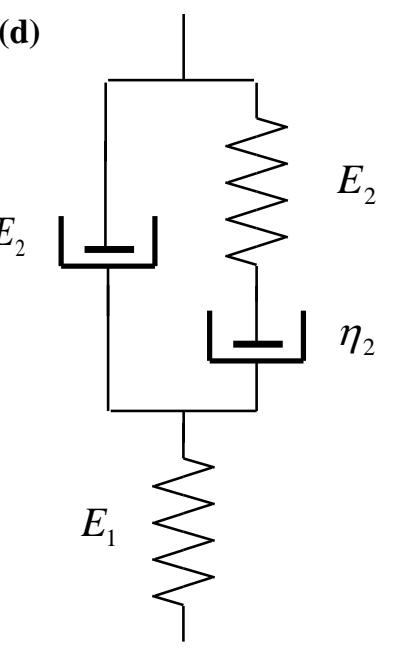

(b)

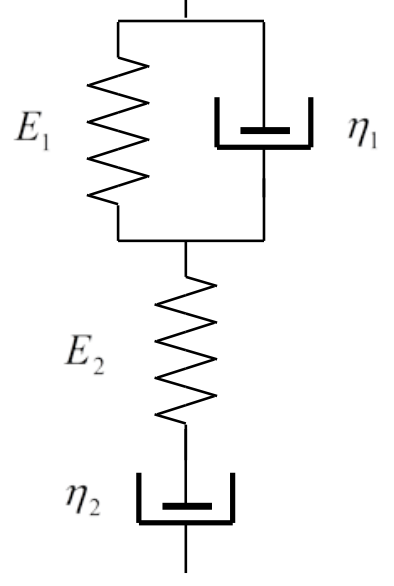

(e)

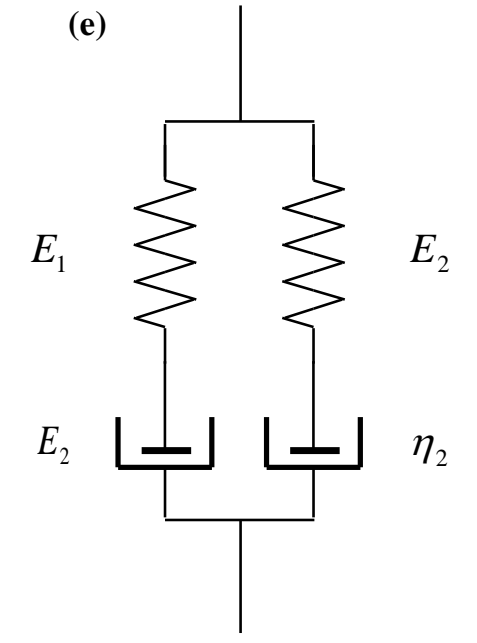

(c)

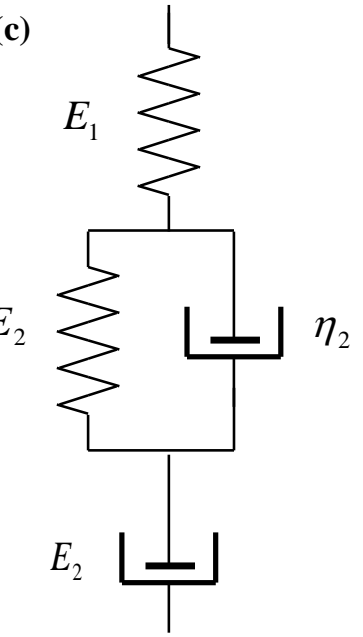


Figure 2

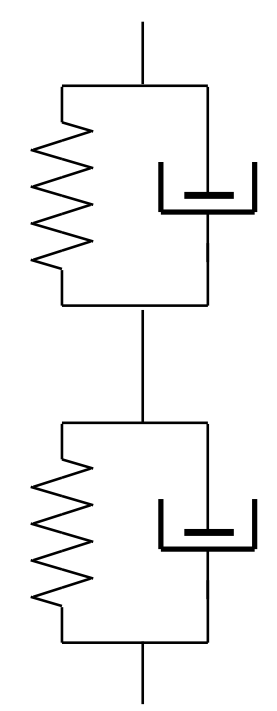


Figure 3

(a)

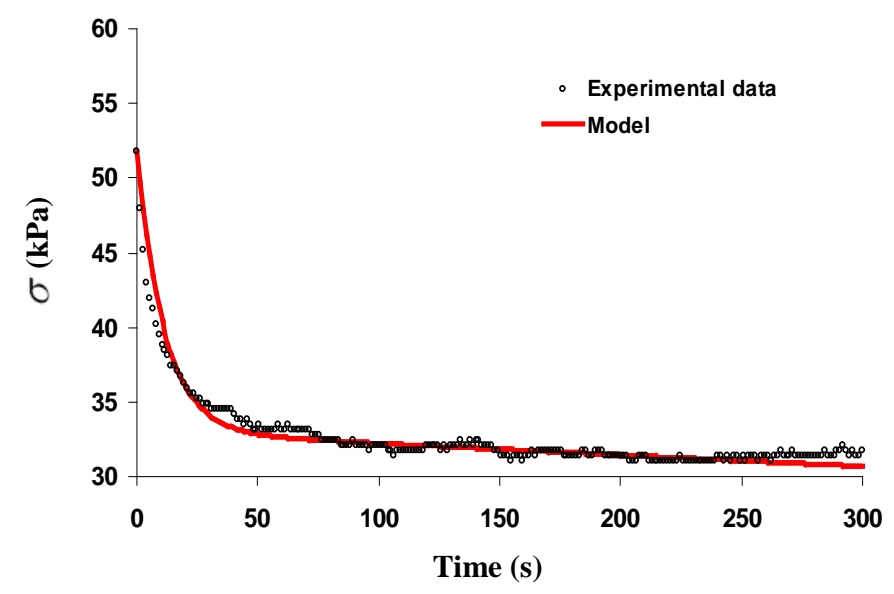

(b)

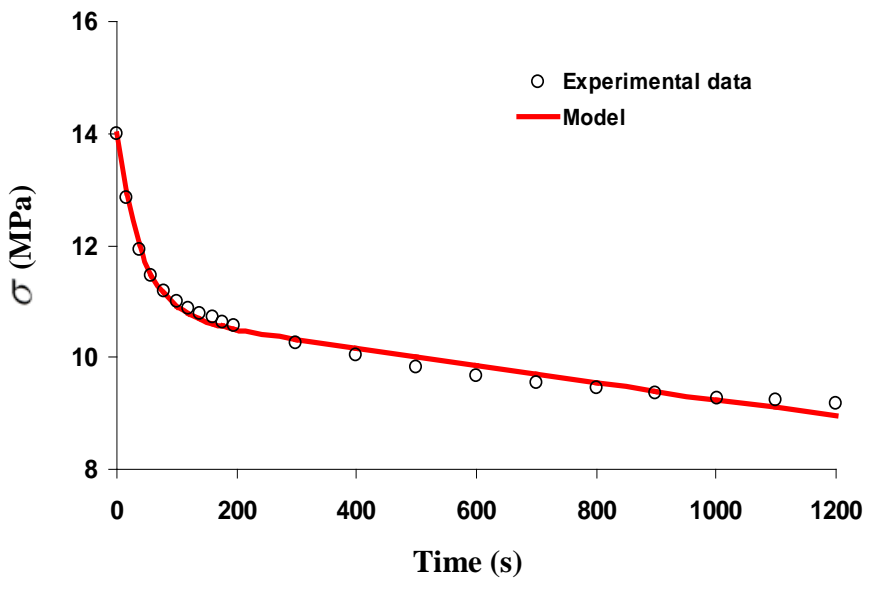

(c)

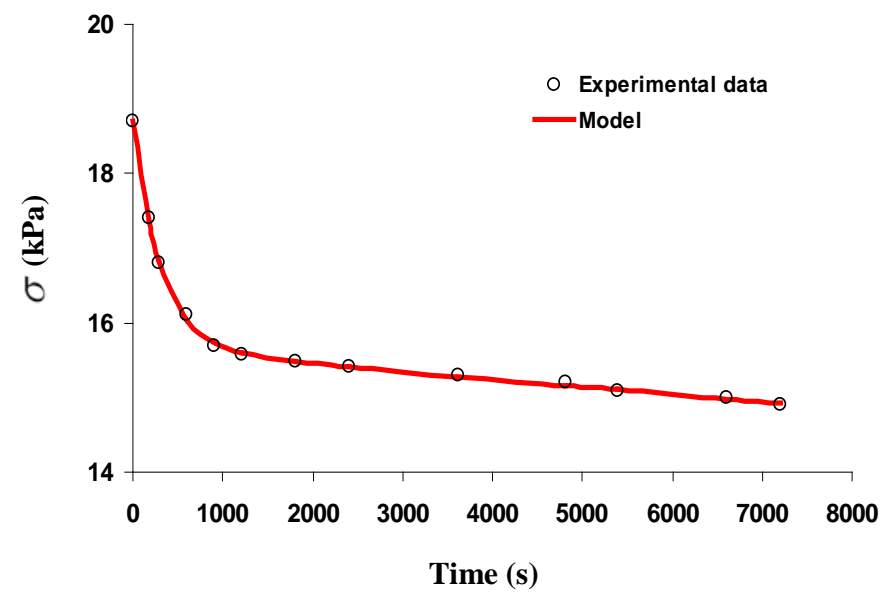

(d)

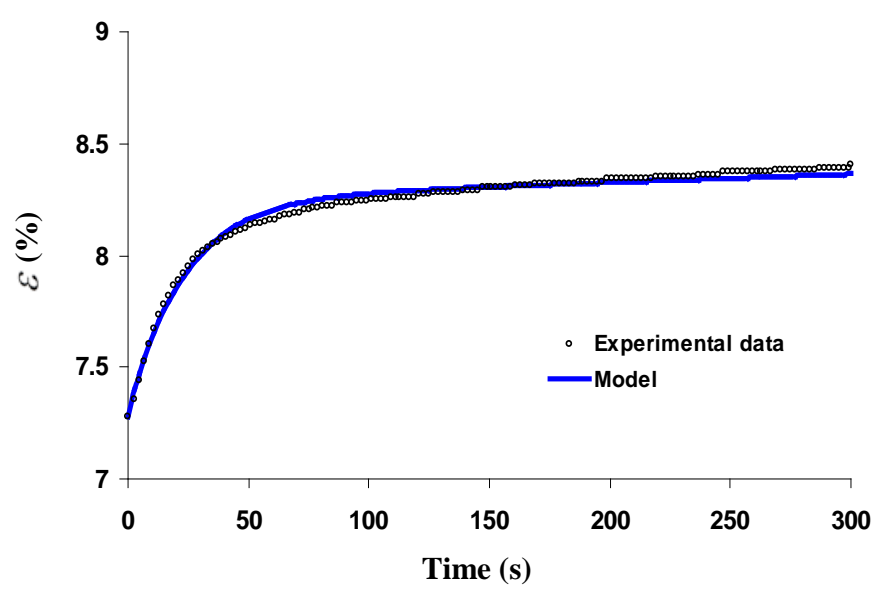

(e)

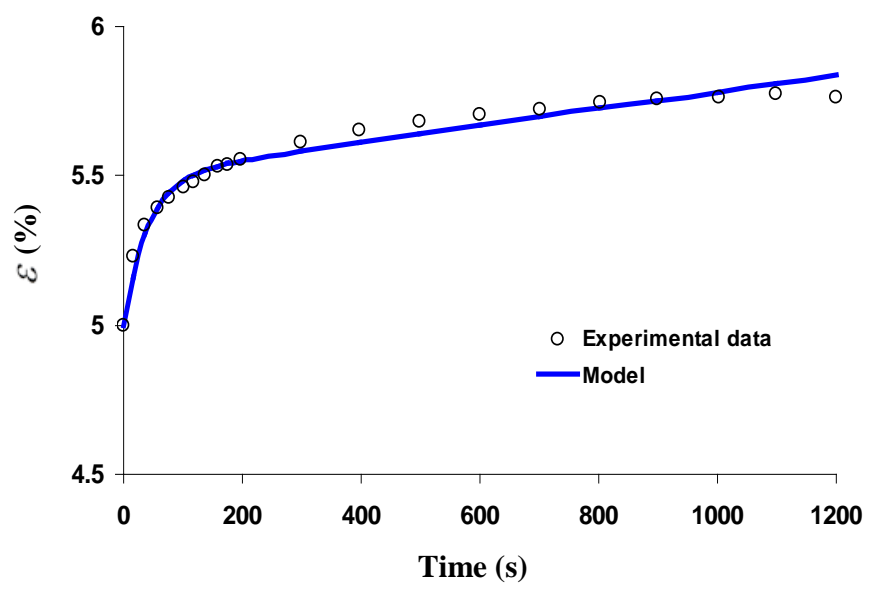

(f)

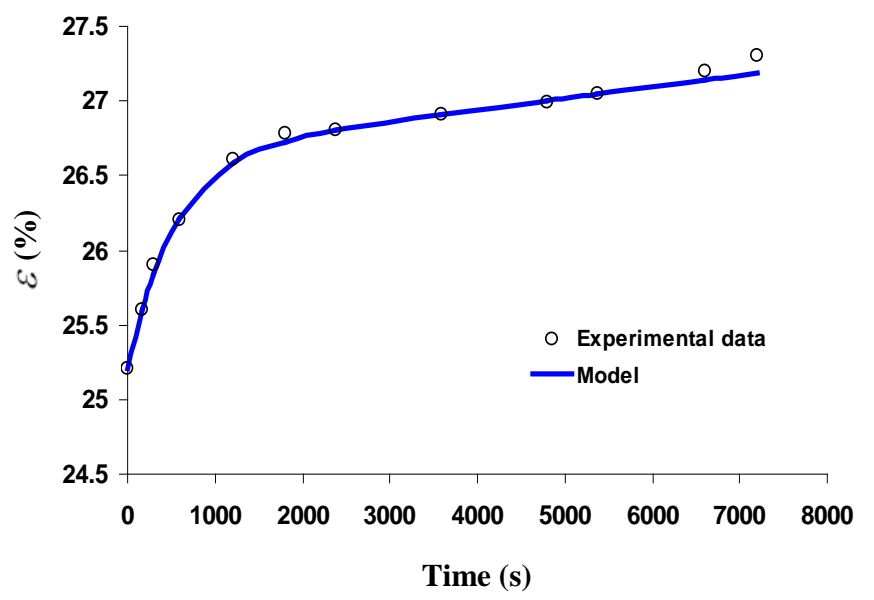

